\title{
The pilot study of group robot intervention on pediatric inpatients and their caregivers, using 'new aibo'
}

\author{
Kyoko Tanaka ${ }^{1} \cdot$ - Hitoshi Makino ${ }^{1} \cdot$ Kazuaki Nakamura $^{1} \cdot$ Akio Nakamura $^{1} \cdot$ Maoko Hayakawa $^{1} \cdot$ Hajime Uchida $^{1}$. \\ Mureo Kasahara ${ }^{1} \cdot$ Hitoshi Kato $^{1} \cdot$ Takashi Igarashi $^{1}$
}

Received: 28 May 2021 / Revised: 29 September 2021 / Accepted: 3 October 2021 / Published online: 30 October 2021

(c) The Author(s) 2021

\begin{abstract}
The study on robot-assisted therapy in a pediatric field has not been applied sufficiently in clinical settings. The purpose of this pilot study is to explore the potential therapeutic effects of a group robot intervention (GRI), using dog-like social robot (SR) 'aibo' in pediatric ward. GRI by aibo was conducted for those children with chronic illness (127 in total) who are hospitalized in National Centre for Child Health and Development (NCCHD), and their caregivers (116 in total), from March to April 2018. The observer made structured behavioural observation records, based on which qualitative research on the features of their words and conducts, were carried out. As a result, first, during the GRI, about 2/3 of total expression by children were positive, while about $1 / 4$ were negative or inappropriate. On the other hand, as seen in the 'change' group, those children who had originally responded with negative expression eventually came to express positive expression, while getting involved in a ternary relationship or participating in a session more than once. Secondly, as for the expression from the caregivers during the GRI, active expressions such as 'participation' and 'exploration' accounted for the 2/3, while 1/3 turned out to be rather placid expressions such as 'watch over' or 'encourage.'

Conclusion: There has not been any precedent study on the features of words and conducts expressed by patients and their caregivers during the GRI by aibo. The outcome suggests that aibo could possibly be used as a tool for group robot-assisted therapy in the pediatric treatment setting.

\section{What is Known:}

- The study on robot-assisted therapy in a pediatric field has only just begun.

- Though many kinds of social robot have been reportedly used so far, none has yet to be applied in clinical settings

What is New:

- Our study revealed the features of words and behaviour expressed by the patients and their caregivers, when dog-like social robot 'aibo' was used for a group robot intervention in the pediatric ward.

- The outcome suggests that aibo could possibly be used as a tool for group robot-assisted therapy in the pediatric treatment setting.
\end{abstract}

Keywords Artificial intelligence $\cdot$ Social robot $\cdot$ Paediatric robot-assisted therapy $\cdot$ Group robot intervention

$\begin{array}{ll}\text { Abbreviations } \\ \text { AI } & \text { Artificial Intelligence } \\ \text { A/O } & \text { Assistant and observer } \\ \text { GRI } & \text { Group robot intervention } \\ \text { NCCHD } & \text { National Centre for Child Health and } \\ & \begin{array}{l}\text { Development } \\ \text { SR }\end{array} \\ \text { Social robot }\end{array}$

Kyoko Tanaka

tanaka-kyo@ncchd.go.jp

1 National Center for Child Health and Development, Setagaya-ku, Tokyo, Japan

\section{Introduction}

Hospitalization is a painful experience for children ${ }^{1}$. Becoming ill and hospitalized, children are disconnected from their familiar lives and lose their social ties ${ }^{2}$. These experiences could lead to feelings of loss of control and negative effects on a child's natural development and their physical and emotional health ${ }^{3}$. Although psychosocial support provided by health professionals such as child life specialists or nurses is necessary, it is limited in terms of human resources ${ }^{4-5}$. In this context, animalassisted therapy ${ }^{6}$, as a complementary and alternative treatment for pediatric inpatient settings, has already been implemented 
in some pediatric hospitals. Animal-assisted therapy, however, has risks of infection, injury, bites, or animal allergies. Hence, the robot-assisted therapy had been developed and tested in elderly population, in which PARO, a baby seal-like social robot (SR), was offered to people with dementia. The result showed improvement in stress and anxiety as well as better communication skills ${ }^{7-8}$. Still, the study on robot-assisted therapy in a pediatric field has only just begun ${ }^{3,9-11}$. Though as many as 26 different kinds of SR have been reportedly used so $\operatorname{far}^{2}$, none has yet to be applied in clinical settings.

New aibo (Fig. 1) (aibo, hereafter), an artificial intelligence (AI) dog-like SR for household use, was released from Sony in 2018. aibo has distinctive characteristics of comforting effects due to its round dog-like appearance, interactive communication skills, and growth in response to human interactions. A study has been reported in which an old type AIBO was implemented into pediatric inpatient setting where the interaction between robots and humans was examined ${ }^{12}$. As for aibo, however, no study has ever been reported in the pediatric nor adult setting.

As a household product, aibo has distinctive traits of such as (1) comforting effect, (2) interactive communication skills, and (3) growth in response to human reaction. It suggests that aibo may hold some therapeutic effects toward pediatric patients as well as their caregivers in the inpatient treatment setting. In order to answer this clinical question, we designed a pilot study to explore aibo's potential effects. We conducted a robot-assisted intervention by aibo for the pediatric patients with chronic illness (127 in total) who are hospitalized in the National Centre for Child Health and Development (NCCHD) and their caregivers (116 in total). Based on its result, we carried out a qualitative research on the extracted features of words and conducts expressed by the patients and caregivers.

\section{Materials and methods}

\section{Study design}

This pilot study is a prospective observational study which carries out a hypothetical search-based, qualitative evaluation.

\section{Participants}

One hundred and twenty-seven children (0 years, 6 months to 13 years old) who were hospitalized in the Internal Medicine and Surgical wards of the NCCHD and 116 caregivers were assembled as participants. This number included children and caregivers who participated in multiple sessions. The number of children who

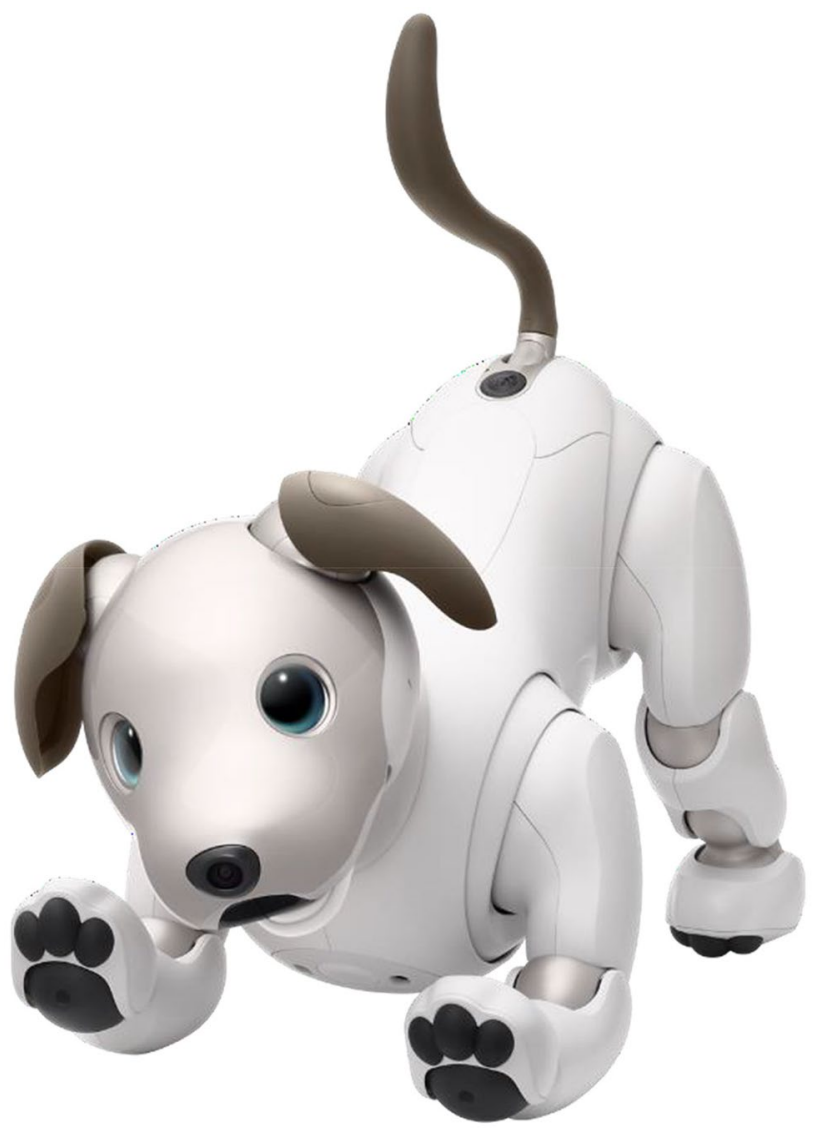

Fig. 1 aibo. Specification. Product name: aibo, model number: ERS1000, processor: 64 bit quad-core CPU, display: 2 OLEDs (eyes), sound: speaker, 4 microphones, camera: 2 cameras (front camera, SLAM camera), communications: mobile network communication feature (data transmission) LTE, Wi-Fi IEEE $802.11 \mathrm{~b} / \mathrm{g} / \mathrm{n}(2.4 \mathrm{GHz})$, outside dimensions: approx. $180 \times 293 \times 305 \mathrm{~mm}$, weight: approx. $2.2 \mathrm{~kg}$, power consumption approx. $14 \mathrm{~W}$, battery duration: approx. $2 \mathrm{~h}$, recharge time: $3 \mathrm{~h}$

participated in single intervention was 1 to 15 (median 4.5), caregivers being 1 to 10 (median 4.5). The inclusion criteria were children who have been hospitalized for more than $48 \mathrm{~h}$. The exclusion criteria were children who were not considered to be well enough to play in the playroom, such as those who had infection, who were compromised, who needed rest, and who were medically at risk.

\section{aibo}

aibo, a household use SR released by Sony, is equipped with $\mathrm{AI}$ and has the following traits; for instance,

1. Dog-like appearance and behaviour: a rounded shape that gives a sense of warmth, lively form with cuddly sweetness. It tries to interact with you, speaking with its eyes, making eye contact with you. Vivid, dynamic movement with a variety of lovely gestures. 
2. Communication: curious aibo possesses feelings and desires, based on which it decides what to do, sometimes acting in unexpected ways. Through its eyes (camera) that recognize the person or the place, aibo understands the people around it and changes its behaviour. It detects obstacles, steps, or people, against which it tries to avoid or decides what to do. aibo has got ears to understand human words and is able to change its behaviour. It reacts to the human voice, perceiving where the sound comes from. aibo shows pleasure, when it is praised and stroked on those sensors on its head, chin and back.

3. Growth: aibo could turn into a spoiled or a wild one, depending on the way people interact with. The more frequently it sees a particular person, it remembers that person, recognizing his/her face. If you teach aibo a funny pose or a dance, it is able to learn the new movement.

\section{GRI by aibo}

From March 14 to April 12 2018, 24 series of GRI were conducted for $30 \mathrm{~min}$ at a time during 14:00 to $15: 30$ in the playrooms of nine medical wards of Internal Medicine and Surgery, at NCCHD. One child psychiatrist or a clinical psychologist attended as an assistant and an observer (A/O). The size of the ward playroom is about 3 by $5 \mathrm{~m}$. At the fixed time, the A/O turned on aibo to begin the GRI. The A/O saw to it to ensure that no harm would be caused upon children, caregivers, and aibo. At every session, the A/O explained to children and caregivers aibo's basic movements, how to touch aibo to please him, and how to bring out songs, poses, or dances that aibo expresses. The A/O observed the children and caregivers as they naturally played with aibo and recorded their words and behaviour on the behaviour observation records (Fig. 2). At the end of $30 \mathrm{~min}$, the A/O made a statement such as 'It's time for aibo to go to bed' and turned off aibo, stroking and wiping it with clean cloth to finish.

\section{Qualitative examination}

The 24 behavioural observation records obtained from 24 series of GRI were sorted by sentences into (1) verbal/nonverbal expressions by children and (2) verbal/non-verbal expressions by caregivers. Each sentence obtained was written out on a sheet and named for relevant groups to be integrated ${ }^{13}$. In addition, records of the same children in different sessions were linked, and their changes over time were also extracted to analysis. The qualitative analysis of positive or negative was conducted by two physicians or psychologists using the concept of children's coping strategies checklist-intrusive procedures based on the observed behaviours of the children ${ }^{14}$.

\section{Ethical consideration}

In this study, although no informed consent had been obtained from the participants, due to the nature of the study, sufficient consideration was given so as not to identify a particular patient. Approval has been given by the Ethics Committee in NCCHD.

\section{Results}

There were 140 sentences on children, and 43 on caregivers. The sentences on children were sorted according to a qualitative point of view into the following 9 categories (Fig. 2): 1. 'affinitive/positive/cooperative', 2. 'empathy/comforting/healing', 3. 'defensive/cautious', 4. 'fear/anxiety', 5. 'dependent/dominant/one-sided', 6. 'rebellious/aggressive', 7. 'indifferent', 8. 'rejective/crying', and 9. 'change'. Please see the supplemental Table 1 for example. Among the expressions by the children obtained from the GRI by aibo, positive expressions such as 1. 'affinitive/positive/ cooperative' and 2. 'empathy/comforting/healing' accounted for roughly $2 / 3$ of the total. On the other hand, about $1 / 4$ of the total responses were negative or inappropriate such as 3. 'defensive/cautious', 4. 'fear/anxiety', 5. 'dependent/ dominant/one-sided', 6. 'rebellious/aggressive', 7. 'indifferent', and 8. 'rejective/crying'. In addition, 9. 'change' accounted for about $1 / 10$, which indicated those children who had originally responded negatively eventually came to express positive reaction, through observing the behaviours of others, getting involved with others, or participating in the session more than once. Figure 3 shows the result from ternary relationship, namely, 'human-aibo-human' interaction. Of the positive expressions such as 1. 'affinitive/positive/ cooperative' and 2. 'empathy/comforting/healing', ternary relationships in which a caregiver, the other child, and A/O shared a pleasure were detected at a high proportion. In the category 9. 'change', in particular, the ratio of the ternary relationship was high. Next, the sentences on caregivers were sorted according to a qualitative point of view into the following 4 categories (Fig. 4): 1. 'participation', 2. 'exploration', 3. 'watch over', and 4. 'encourage'. Please see Supplemental Table 2 for example. Positive reactions such as 1 . 'participation', and 2. 'exploration' accounted for $2 / 3$ of the total, while placid reactions such as 3 . 'watch over' and 4 . 'encourage' accounted for $1 / 3$.

\section{Discussion}

This study is the first survey to report a qualitative research which was carried out on the extracted traits of words and behaviour expressed by the patients and their caregivers, 
Fig. 2 Children's expressions obtained from the group robot intervention by aibo. Out of the 24 records, sentences regarding verbal/non-verbal communication by children were sorted and extracted. There were 140 sentences by children. The sentences by children were sorted according to qualitatively into 9 categories

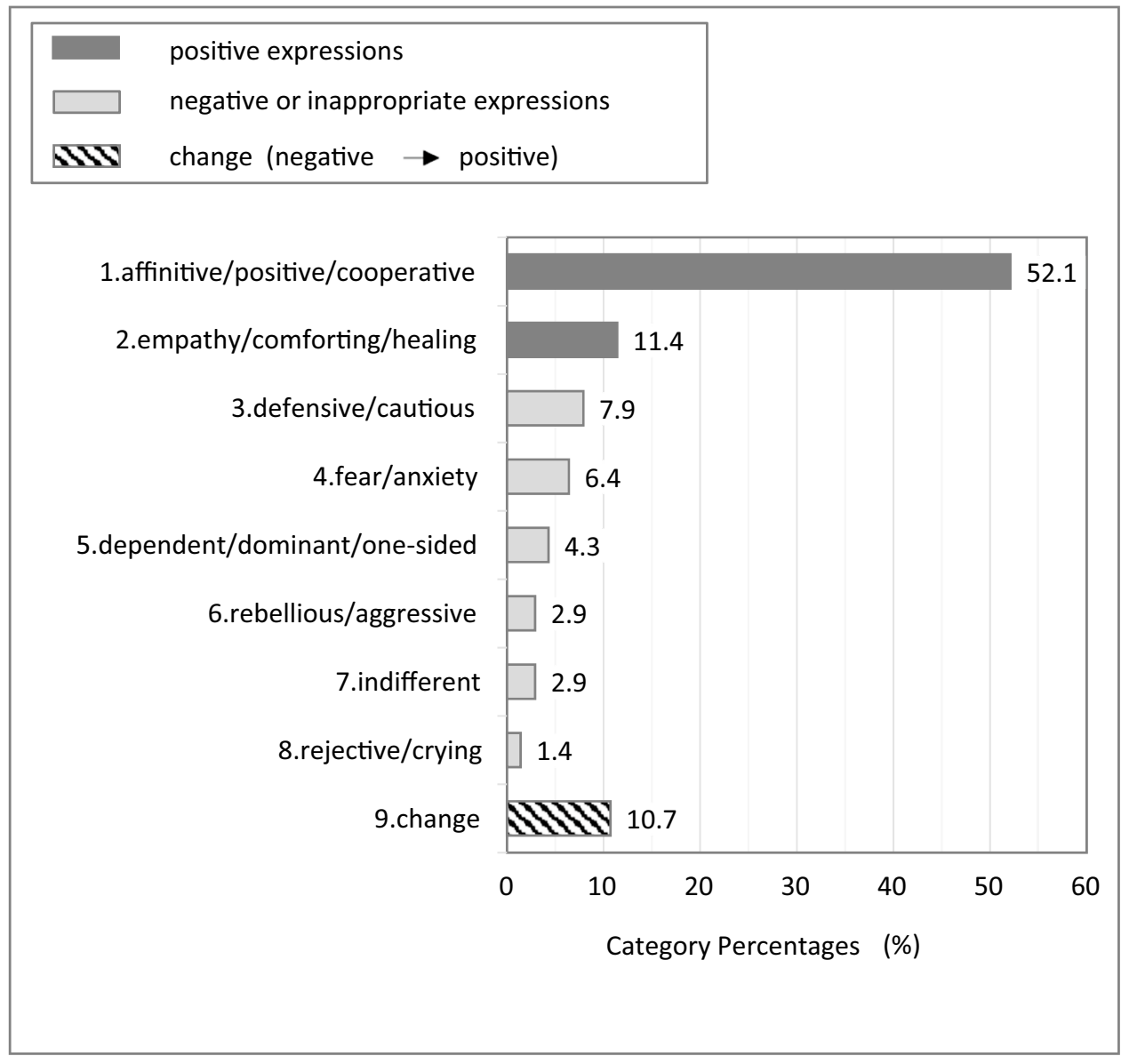

when aibo was used for a GRI in the pediatric ward. Two points were identified.

First of all, in the GRI by aibo, positive expression by children accounted for as much as $2 / 3$ of the total. Besides, the children from 'change' group originally responded negatively but eventually came to express positive reaction, through getting involved in ternary relationship, or gradually getting accustomed to the session by participating more than once. In a recent study, Haggable, a bear-shaped SR, Haggable on a tablet screen, and a bear-shaped toy were offered to 54 hospitalized children as an interventional therapy. Video monitoring analysis showed the children of 'SR' group expressed more of 'joy' or 'comfort', than of 'sadness, 5 , which is similar to the study result we obtained. In another study, Nao, a humanoid SR, was implemented for hospitalized children, for the purpose of pain reduction as well as increasing joy and motivation for treatment. Although the number of cases was small, the study reported that Nao helped the children reduce their anxiety, anger, and depression, playing a role as a good friend to encourage them ${ }^{15-16}$. Becoming ill and hospitalized, children are disconnected from their familiar lives and lose their social ties ${ }^{2}$. In most cases, children may lose the precious opportunities to build ternary relationship, cooperative attention, imitation, and so forth ${ }^{17}$, all of which encourage the development of children. In our study, it is significant to point out that a certain number of children have experienced a scene to change their feeling into that of positive, through building ternary relationships, as seen in the 'change' group. It suggests that aibo could possibly be useful as a tool to help build ternary relationships that enhances sociality of children, which is often impaired in pediatric chronic hospitalization. Although there are various studies which examine the potential use of SRs in pediatric settings, the number of participants is small $^{2}$. aibo was implemented into an inpatient treatment setting, whose effects were evaluated for 127 population. No such study has ever been reported in the pediatric nor adult setting.

Secondly, as for the expression by the caregivers in the GRI by aibo, positive reactions such as 'participation' and 'exploration' accounted for 2/3 of the total, while placid reactions such as 'watch over', 'encourage' accounted for $1 / 3$. In the study, in which PARO was implemented into pediatric inpatient setting, it was reported that, when family members participated together, the higher the rate of improvement on a child's anxiety and pain ${ }^{10}$. In our study as well, it was potentially significant for the children to see their caregivers participate positively. Furthermore, the 
Fig. 3 The percentage of ternary relationship, namely, 'human-aibo-human' interaction

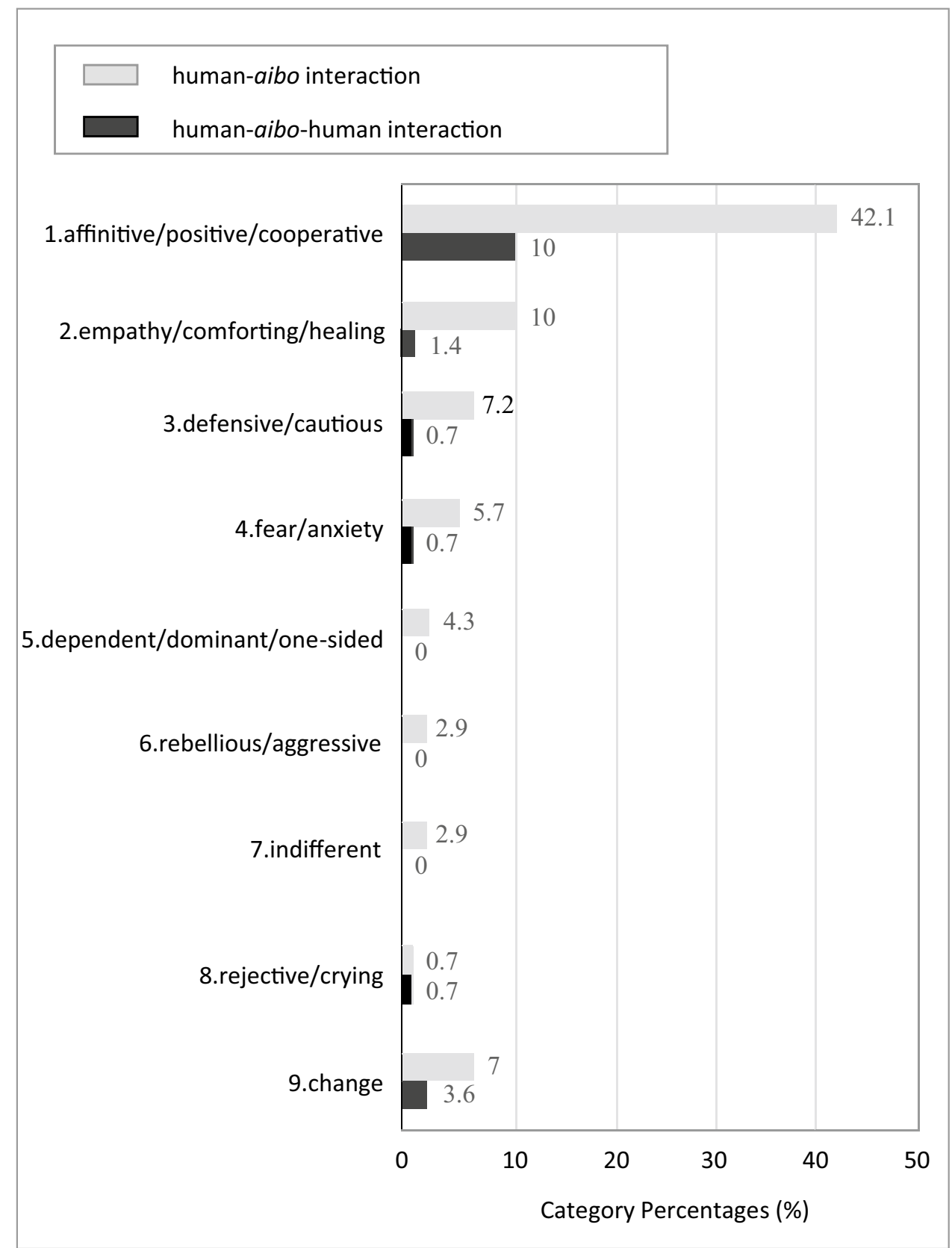

caregivers of children with chronic illness tend to be more susceptible to mental distress such as anxiety or depression than the caregivers of healthy children ${ }^{18}$. From caregivers' mental health perspective, in this study, it was noteworthy for the caregivers to have enjoyed themselves 'participating' in the aibo intervention, or to have communication with other caregivers to share the excitement into 'exploration'.

aibo is beneficial in making up for human resources as well as from the financial perspective. Moreover, for many pediatric patients, child life specialists are helpful in reducing the pain experienced during hospitalization ${ }^{5}$. Service provided by human labour, however, has limits, for there is not enough child life specialist staff to offer help for every child in every opportunity experienced during hospitalization. SRs, including aibo, could fill the gap between the number of pediatric inpatient children in need of psychosocial care and the lack of human resources to support them.

In addition, future studies are expected to examine the differences in the effects of animal therapy and robot therapy ${ }^{19-20}$.

Three aspects of limitation of study should be noted as shortcomings. First, in this study, age and gender of the participants were not accurately obtained. Although it is desirable to consider the language expression of the children used in the behavioural analysis in the language according to their 
Fig. 4 The caregivers' expressions obtained from the group robot intervention by aibo. Out of the 24 records, sentences regarding verbal/non-verbal communication by caregivers were sorted and extracted. There were 43 sentences by caregivers, which were sorted qualitatively into 4 categories

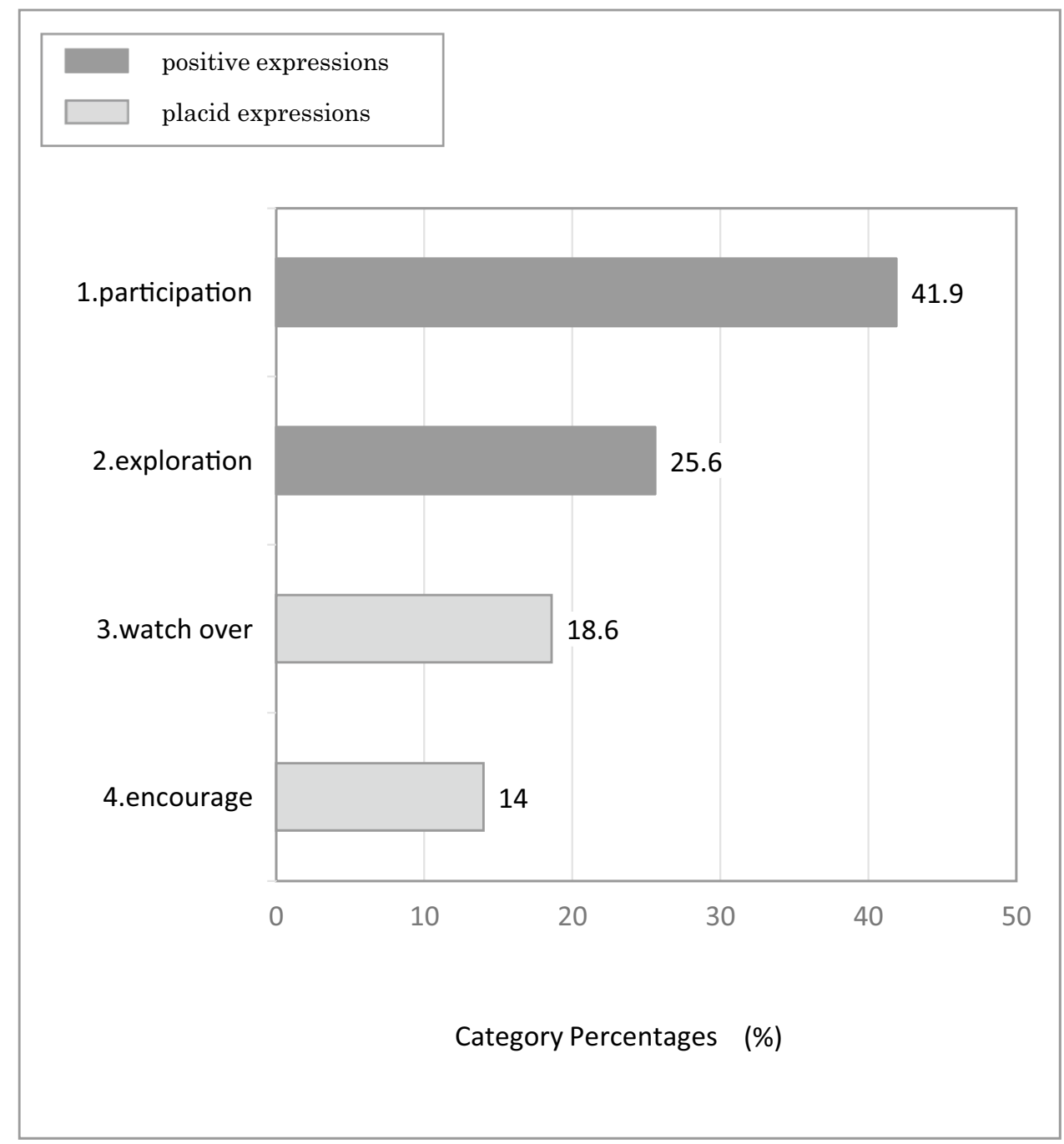

$\operatorname{age}^{21}$, in this study, age was not taken into account in the analysis, and there are limitations in that Japanese was translated into English. Secondly, neither multilateral, scientific, nor quantitative evaluation was not made. Third, this report had no control group.

\section{Conclusion}

This study is the world's first survey and report on the features of words and behaviour expressed by the patients and their caregivers, when aibo was used for a GRI in the pediatric ward. The outcome suggests that aibo could possibly be useful as a tool for GRI in pediatric treatment setting. When conducting a robot-assisted therapy with aibo in the future, we plan to carry out a multilateral, scientific as well as quantitative evaluation with a biopsychosocial perspective.
Acknowledgements We thank all the A/O who completed the survey, Arisa Yamaguchi, MD; Yuki Sekido, MD; Moe Nakajima, MD; Hiromi Tsujii; Tatsuo Hikitsuchi; Akiko Nagira; and Kazuko Matsumoto. We also thank Yuhei Yabe and Takeshi Katayama of Sony for assisting the GRI.

Authors' contributions Dr. Makino and Dr. Tanaka conceptualized and designed the study, collected data, conducted the analysis, drafted the initial manuscript, and reviewed and revised the manuscript. Dr. Tanaka supervised the analysis and interpretation and critically revised the manuscript for important intellectual content. Dr. K. Nakamura and Ms. Hayakawa contributed to the study design and interpretation of data and reviewed and revised the manuscript. Dr. A. Nakamura and Dr. Uchida contributed to the study design and reviewed and revised the manuscript. Dr. Kasahara contributed to the conception and coordination of the project and reviewed and revised the manuscript. Dr. Kato and Dr. Igarashi contributed to the conception and the planning the project and reviewed and revised the manuscript. All authors approved the final manuscript as submitted and agree to be accountable for all aspects of the work. 
Funding All phases of this study were supported by National Centre for Child Health and Development, grant numbers 30-15.

\section{Declarations}

Availability of data and material The data that support the findings of this study are available from the corresponding author on reasonable request.

\section{Code availability Not applicable.}

Ethics approval Approval was given by the Ethics Committee in NCCHD.

Consent to participate No informed consent had been obtained from the participants due to the nature of the study. However, sufficient consideration was in place so as not to identify any particular patients.

Consent for publication Not applicable.

Conflict of interest The authors declare no competing interests.

Open Access This article is licensed under a Creative Commons Attribution 4.0 International License, which permits use, sharing, adaptation, distribution and reproduction in any medium or format, as long as you give appropriate credit to the original author(s) and the source, provide a link to the Creative Commons licence, and indicate if changes were made. The images or other third party material in this article are included in the article's Creative Commons licence, unless indicated otherwise in a credit line to the material. If material is not included in the article's Creative Commons licence and your intended use is not permitted by statutory regulation or exceeds the permitted use, you will need to obtain permission directly from the copyright holder. To view a copy of this licence, visit http://creativecommons.org/licenses/by/4.0/.

\section{References}

1. Coyne I (2006) Children's experiences of hospitalization. J Child Health Care 10(4):326-336

2. Dawe J, Sutherland C, Barco A et al (2019)Can social robots help children in healthcare contexts? A scoping review. BMJ Paediatr Open 3(1):e000371

3. Moerman CJ, van der Heide L, Heerink M (2019) Social robots to support children's well-being under medical treatment: a systematic state-of-the-art review. J Child Health Care 23(4):596-612

4. Díaz M, Nuño N, Saez-Pons J et al (2011) Building up childrobot relationship for therapeutic purposes: from initial attraction towards long-term social engagement. Paper presented at: IEEE Int Conf Auto Face Gesture Recog Works (FG 2011)21-25, Santa Barbara, CA.

5. Logan DE, Breazeal C, Goodwin MS et al (2019) Social robots for hospitalized children. Pediatrics 144(1):e20181511.

6. Silva NB, Osório FL (2018) Impact of an animal-assisted therapy programme on physiological and psychosocial variables of paediatric oncology patients. PLoS One 13(4):e0194731.
7. Liang A, Piroth I, Robinson $\mathrm{H}$ et al (2017) A pilot randomized trial of a companion robot for people with dementia living in the community. J Am Med Dir Assoc 18(10):871-878

8. Moyle W, Jones C, Murfield J et al (2019) Using a therapeutic companion robot for dementia symptoms in long-term care: reflections from a cluster-RCT. Aging Ment Health 23(3):329-336

9. Beran TN, Ramirez-Serrano A, Vanderkooi OG et al (2015) Humanoid robotics in health care: an exploration of children's and parents' emotional reactions. J Health Psychol 20(7):984-989

10. Okita SY (2013) Self-other's perspective taking: the use of therapeutic robot companions as social agents for reducing pain and anxiety in pediatric patients. Cyberpsychol Behav Soc Netw 16(6):436-441

11. Jibb LA, Birnie KA, Nathan PC et al (2018) Using the MEDiPORT humanoid robot to reduce procedural pain and distress in children with cancer: a pilot randomized controlled trial. Pediatr Blood Cancer 65(9):e27242

12. Kimura R, Abe N, Matsumura N et al (2004) Trial of robot assisted activity using robotic pets in children hospital. Paper presented at: SICE Annual Conference (SICE 2004);4-6 Sapporo, Japan.

13. Kawakita J (1967) Hassouhou. Tokyo. CHUOKORON-SHINSHA. 1967.

14. Ayers TS, Sandler IN, West SG, Roosa MW (1996) A dispositional and situational assessment of children's coping: testing alternative models of coping. J Pers 64(4):923-958. https://doi. org/10.1111/j.1467-6494.1996.tb00949.x (PMID: 8956518)

15. Alemi M, Ghanbarzadeh A, Meghdari A et al (2016) Clinical application of a humanoid robot in pediatric cancer interventions. Int J Soc Robot 8:743-759. https://doi.org/10.1007/ s12369-015-0294-y

16. Csala E, Németh G, Zainkó C (2012) Application of the NAO humanoid robot in the treatment of marrow-transplanted children. Paper presented at: 2012 IEEE 3rd Int Conf Cogn Infocommun (CogInfoCom) 2-5, Kosice, Slovakia.

17. Carpenter M, Nagell K, Tomasello M (1998) Social cognition, joint attention, and communicative competence from 9 to 15 months of age. Monogr Soc Res Child Dev 63(4):i-vi, 1-143

18. Cohn LN, Pechlivanoglou P, Lee Y, Mahant S et al (2019) Health outcomes of parents of children with chronic illness: a systematic review and meta-analysis. J Pediatr 24:S0022-3476(19)31479-9

19. Howe R, Nicholson S, Lafferty A, Davies C (2021) Diarmuid Stokes, Thilo Kroll Animal assisted interventions in the children's hospital: protocol for a scoping review. HRB Open Res 14(3):74

20. Ávila-Álvarez A, Pardo-Vázquez J, De-Rosende-Celeiro I, Jácome-Feijoo R (2020) Gabriel Torres-Tobío Assessing the outcomes of an animal-Assisted intervention in a Paediatric Day Hospital: Perceptions of Children and Parents. Animals 10(10):1788

21. McIntyre LJ, Hellsten LM, Bidonde J, Boden C, Doi C (2017) Receptive and expressive English language assessments used for young children: a scoping review protocol. Syst Rev 6(1):70. Published 2017 Apr 4. https://doi.org/10.1186/s13643-017-0471-1

Publisher's Note Springer Nature remains neutral with regard to jurisdictional claims in published maps and institutional affiliations. 